\section{Hot off the breath: Mortality as a primary end-point in IPF treatment trials: the best is the enemy of the good}

The problem of the selection of accurate primary end-points for treatment studies in idiopathic pulmonary fibrosis (IPF) has recently been aired in a controversial paper from the USA. ${ }^{1}$ The limitations of current end-points are discussed and the authors conclude that all-cause mortality and all-cause nonelective hospitalisation best meet clinically meaningful end-point criteria. Much of the article is well argued and there is no quarrel with the view that current primary end-points are flawed. We also agree that all-cause mortality would, indeed, be the most clinically meaningful primary end-point and, therefore, the preferred primary end-point, were it not impractical, as discussed below. However, readers of the statement should reflect on the wise maxim that 'the best may be the enemy of the good'. The purpose of our document is to provide a perspective on all-cause mortality as a primary endpoint, endorsed by 52 European clinicians Including the authors (with one abstention), exploring the implications of the statement by Raghu and colleagues. We believe strongly that the adoption of the views of these authors by licensing bodies -with, by implication, a statistically significant mortality benefit a pre-requisite for drug registration-would set back

\footnotetext{
${ }^{1}$ Department of Respiratory Medicine, Royal Brompton Hospital, London, UK

${ }^{2}$ Department of Internal Medicine III, Respiratory and Critical Care, University Hospital Bergmannsheil, Bochum, Germany

${ }^{3}$ Medical Faculty, University of Duisburg-Essen, and Ruhrlandklinik, Essen, Germany

${ }^{4}$ Service de Pneumologie, Hôpital Louis Pradel, Lyon, France

${ }^{5}$ Department of Respiratory Medicine, PierantoniMorgagni Hospital, Forlì, Italy

${ }^{6}$ Centre for Rare Lung Diseases, University of Modena Hospital, Modena, Italy
}

Correspondence to Athol U Wells, C/O Emmanuel Kaye Building Manresa Road, Chelsea, London SW3 6LR, UK; athol.wells@rbht.nhs.uk progress in the treatment of IPF by a decade or more.

It should be acknowledged at the outset that the statement of Raghu and colleagues does not make explicit recommendations with regard to drug licensing. Indeed, the authors declare that it is not their aim to make such recommendations and their intentions in this regard should not be questioned. However, if the statement has, indeed, been widely 'misread', the reasons for this are clear enough. Representatives of the US Food and Drug Administration (FDA) were active participants in a forum in Bethesda, Maryland (July 2011) which gave rise to the document as a proceedings statement. ${ }^{1}$ It is widely known that if current phase three studies (of either pirfenidone or nintedanib (BIBF 1120)) show a positive treatment effect on serial change in forced vital capacity (FVC), registration applications will follow in the near future. As recently as a year ago, all but two of the authors endorsed the view that in IPF 'a change in absolute FVC of $10 \% \ldots$ is a surrogate marker of mortality and is evidence of, in the absence of an alternative explanation, disease progression'.2 Given the apparent volte-face in the current document, the timing of the statement and the participation by FDA personnel, there is a genuine risk that the registration process will, indeed, be influenced.

The heart of the matter is the stated view that because a phase three IPF study (the INSPIRE study) provided mortality non-efficacy signal within a realistic inter$\mathrm{val}^{3}$ and significant mortality differences were observed in another phase three IPF study (the PANTHER study), ${ }^{4}$ treatment trials with mortality as a primary endpoint are, in general, feasible. The same conclusion might, in principle, be drawn from findings in the recently published placebo-controlled evaluation of warfarin therapy in $\mathrm{IPF}^{5}$ in which active treatment was associated with a statistically significant increase in mortality.

However, the fatal flaw in this reasoning is the fact that in none of these studies was efficacy demonstrated. In the PANTHER and warfarin studies, an early adverse effect was observed which, in the PANTHER study, appeared to be largely confined to the first 4 months of treatment. Although the numbers of deaths in both studies were low (an expected consequence of the short study duration), the proportionate increase in mortality was striking, when compared with the placebo arm. From these observations it can be concluded that if a striking early harmful effect is associated with a studied intervention, an early mortality signal should, indeed, be expected. It appears intrinsically likely that a placebocontrolled trial of cyanide therapy in IPF would deliver an early and definitive result. If there is an obvious lack of efficacy, as in the INSPIRE study, a longer study period will be required but a conclusion based on mortality data can be anticipated in a realistically short time interval (provided that studies are powered for a major and possibly unrealistic reduction in mortality).

However, when treatment efficacy (as opposed to futility or harm) is considered, a very different picture emerges. The problem, here, is the consistently low mortality rate in placebo arms of recent treatment studies, possibly due in part to the selective enrolment of patients with less progressive disease in placebo-controlled trials. A $25 \%$ reduction in mortality would be widely regarded as a major treatment benefit, given the likelihood that treatment advances in IPF will consist of small incremental benefits. It has been estimated, based on the observed mortality in the recent placebo arms of IPF studies, that for a $25 \%$ reduction in mortality to be statistically significant in a placebo-controlled IPF trial, the enrolment of 2600 patients and 5 years of follow-up would be required. ${ }^{6}$

We believe that the performance of such a study is impracticable. Currently, pirfenidone, licensed as an IPF treatment in Europe, can be prescribed in eight European countries and is available through named patient programmes in a number of others. Anti-oxidant therapy is also widely prescribed in patients wishing for active treatment. Based on the pirfenidone precedent, European registration of nintedanib is likely to be pursued, in the event of a positive result in the current nintedanib phase three studies. In the USA, enrolment is 
currently underway in a number of interventional studies. It should be stressed that the current availability of open treatments and interventional studies contrasts greatly with the lack of treatment alternatives at the time of enrolment in the INSPIRE study. It is unlikely, given current opportunities, that patients motivated to take part in IPF treatment studies, will accept a protocol which requires a commitment to long-term treatment, despite obvious progression of disease (whether due to non-efficacy or to enrolment in a placebo arm). It is surely far more probable that when progression is evident, many patients will opt for open treatment or enrolment in an alternative treatment study. Raghu and colleagues would not countenance efforts to dissuade patients from seeking alternatives in the event of obvious treatment failure, but given this fact, a protocol requiring lifelong placebo-controlled treatment seems unrealistic.

Nor can it be expected that useful mortality efficacy data might arise from a less definitive approach. For example, it cannot be argued with credibility that concordant mortality trends should be required in phase three studies in order to 'validate' statistically significant beneficial treatment effects on other end-points. In current phase three studies, in which a harmful treatment effect is not evident, a very low mortality rate is necessarily associated with wide CIs. The low number of deaths dictates that chance alone is likely to give rise to much larger proportionate variations in mortality than, for example, a hoped for $25 \%$ reduction in death rate.

The performance of mortality studies in advanced IPF might seem to be an attractive option but this approach, also, is problematic. Patients enrolled in the placebo-controlled STEP study of sildenafil, an agent with no proven clinically significant anti-fibrotic activity, were considered to have severe IPF. ${ }^{7}$ The observed treatment effects on gas transfer and $\mathrm{pO} 2$ levels are widely regarded as indicative of a beneficial effect on the pulmonary vessels. However, if so, this underlines the impact of pulmonary hypertension in advanced IPF, with a high likelihood that death from pulmonary hypertension will confound any mortality benefit from an anti-fibrotic agent in this patient sub-group.

A further concern arising from a requirement for mortality data for drug registration in IPF is the likely effect on evaluation of future novel therapies. The exponential increase in the last decade in the number of patients enrolled in trials of IPF treatments is largely ascribable to the pharmaco-economic model of drug development. The USA IPF clinical research network (IPFnet) should be commended for their admirable work in examining several currently available treatments, including warfarin, sildenafil, anti-oxidant therapy and 'triple therapy'. However, the successful introduction of novel agents demands a level of investment that lies far beyond the scope of state-sponsored initiatives. A business model of drug development is required for this purpose. A requirement for prolonged phase three mortality studies of large IPF patient populations as a prerequisite for drug registration, at an estimated cost of US \$250 million, ${ }^{6}$ would inevitably deter and might entirely prevent pharmaceutical companies from developing novel treatments for IPF.

For all of these reasons, we believe strongly that serial trends in FVC remain the preferred primary end-point in treatment studies, as recently argued by an expert group. ${ }^{8}$ The limitations of FVC should be acknowledged. Uncertainties exist regarding the optimal threshold for 'significant change" and, importantly, trends in FVC and other objective variables are not synonymous with changes in dyspnoea, health status or quality of life. ${ }^{10-13}$ All measures of outcome in IPF are flawed but this truism, stressed by Raghu et $a l,{ }^{1}$ is equally applicable to outcome variables used in all other chronic disorders. The limitations of the 6 min walk test are widely acknowledged and yet, with its use as a primary endpoint in treatment studies, the therapeutic landscape has been transformed in pulmonary arterial hypertension (PAH). It is sobering to reflect that, had mortality data been required for drug registration, it is highly unlikely that targeted $\mathrm{PAH}$ therapies would now be available. The same is true in cystic fibrosis (CF), where mortality has long been abandoned as a trial end-point. In IPF, the advantages of FVC over the 6 min walk distance and other candidate end-points include excellent measurement characteristics and a consistent linkage between categorical FVC changes and mortality ${ }^{9}$ 14-19 - and, also, other clinically important variables. ${ }^{19}$

The view that the prevention of disease progression is not a worthy primary goal, in its own right, seems counterintuitive, if not perverse. We acknowledge that the use of FVC trends primarily as a surrogate for mortality (as opposed to a marker of disease progression) can be questioned.
Raghu and colleagues cite a rigorous definition by which validation of a surrogate requires that changes in the surrogate are associated with subsequent concordant changes in mortality. ${ }^{20}$ The concern, here, for which there is precedence, ${ }^{21}$ is the possibility that a treatment might have a beneficial effect on a surrogate but an adverse effect on mortality. However, this possibility is surely best excluded by a requirement for pharmaceutical companies to provide long-term open treatment data, including mortality data, after trial completion. Although less definitive, this approach is, at least, possible. Treatment data, validating surrogates of mortality against subsequent mortality, exist in only a handful of diseases with a high prevalence and are highly unlikely to emerge in IPF.

In summary, we are vehemently opposed to the idea that mortality data should be required for drug registration in IPF. We view mortality as a highly unsuitable primary end-point in phase three trials: unacceptable to patients and likely to deter pharmaceutical companies from developing new IPF therapies. Mortality data are not required for drug registration, whether in rare respiratory diseases such as $\mathrm{PAH}$ and $\mathrm{CF}$ or in common respiratory diseases such as lung cancer and chronic obstructive pulmonary disease, and the idea that this should be required in IPF is an offence against natural justice and our patients.

Collaborators Endorsed by Albera C, Ancochea J, Antoniou KM, Bonella F, Bonniaud P, Bouros D, Bresser $P$ Cordier JF, Crestani B, Domagala-Kulawik J, Drent M, Egan JJ, Geiser T, Grunewald J, Grutters J, Gudmundsson G, Guenther A, Harari S, Harrison NK, Hirani N, Hodgson U, Kahler CM, Keane MP, Kiter G, Kneussl M, Maher TM, Mogulkoc N,Muller-Quernheim $J$, Neurohr C, Nunes H, Parfrey H, Peros-Golubicic T, Polychronopoulos V, Prevot G, Renzoni ER, Robalo Cordeiro C, Saltini C, Skold M, Spagnolo P, Thomeer M, Tomasseti S, Valeyre D, Vancheri C, Wallaert B, Wuyts W, Xaubet A

Contributors All authors contributed to the content and drafting of this manuscript. In addition, we list 34 European collaborators on the authorship page.

Provenance and peer review Commissioned; internally peer reviewed.

Received 16 August 2012

Accepted 17 August 2012

Thorax 2012;67:938-940.

doi:10.1136/thoraxjnl-2012-202580

\section{REFERENCES}

1. Raghu G, Collard HR, Anstrom KJ, et al. Idiopathic pulmonary fibrosis: clinically meaningful primary end-points in phase 3 clinical trials. Am J Respir Crit Care Med 2012;185:1044-8.

2. Raghu G, Collard HR, Egan JJ, et al. An official ATS/ ERS/JRS/ALAT statement: idiopathic pulmonary fibrosis: evidence-based guidelines for diagnosis and 
management. Am J Respir Crit Care Med 2011;183:788-824.

3. King TE Jr, Albera C, Bradford WZ, et al. Effect of interferon gamma-1b on survival in patients with idiopathic pulmonary fibrosis (INSPIRE): a multicentre randomised placebo-controlled trial. Lancet 2009;374:222-8

4. Raghu G, Anstrom KJ, King TE Jr, et al., Idiopathic Pulmonary Fibrosis Clinical Research Network. Prednisolone, azathioprine and N-acetylcysteine for pulmonary fibrosis. $N$ Engl J Med 2012;366:1968-77.

5. Noth I, Anstrom KJ, Calvert SB, et al. A placebo-controlled randomized trial of warfarin in idiopathic pulmonary fibrosis. Am J Respir Crit Care Med 2012;186:88-95.

6. Bradford WZ, Cohen AH, Leff JA. Selection of clinically meaningful primary end-points in phase 3 clinical trials in idiopathic pulmonary fibrosis (letter). Am J Respir Crit Care Med 2012; in press.

7. Zisman DA, Schwarz M, Angstgrom KJ, et al., (Idiopathic Pulmonary Fibrosis Clinical Research Network). A controlled trial of sildenafil in advanced idiopathic pulmonary fibrosis. N Engl J Med 2010;363:620-8.

8. du Bois RM, Nathan SD, Richeldi L, et al. Idiopathic pulmonary fibrosis: lung function is a clinically meaningful endpoint for phase 3 trials. Am J Respir Crit Care Med Published Online First: 12 July 2012. doi: 10.1164/rccm.201206-1010PP

9. Richeldi L, Ryerson CJ, Lee JS, et al. Relative versus absolute change in forced vital capacity in idiopathic pulmonary fibrosis. Thorax 2012;67:407-11.

10. Yorke J, Jones PW, Swigris JJ. Development and validity testing of an IPF-specific version of the St George's Respiratory Questionnaire. Thorax 2010;65:921-6.

11. Patel AS, Siegert RJ, Brignall K, et al. The development and validation of the King's Brief Interstitial Lung Disease (K-BILD) health status questionnaire. Thorax 2012; 67: 804-10.

12. De Vries J, Kessels BL, Drent M. Quality of life of idiopathic pulmonary fibrosis patients. Eur Respir $\mathrm{J}$ 2001;17:954-61.

13. Behr J. Mortality, a bar too high for IPF clinical trials! (letter). Am J Respir Crit Care Med; in press.

14. Latsi PI, du Bois RM, Nicholson AG, et al. Fibrotic idiopathic interstitial pneumonia: the prognostic value of longitudinal functional trends. Am J Respir Crit Care Med 2003:168:531-7.

15. Collard HR, King TE Jr, Bartelson BB, et al. Changes in clinical and physiologic variables predict survival in idiopathic pulmonary fibrosis. Am J Respir Crit Care Med 2003;168:538-42.

16. Flaherty KR, Mumford JA, Murray S, et al. Prognostic implications of physiologic and radiographic changes in idiopathic interstitial pneumonia. Am J Respir Crit Care Med 2003;168:543-8.

17. King TE Jr, Safrin S, Starko KM, et al. Analyses of efficacy end points in a controlled trial of interferon-gamma1b for idiopathic pulmonary fibrosis. Chest 2005;127:171-7

18. Jegal Y, Kim DS, Shim TS, et al. Physiology is a stronger predictor of survival than pathology in fibrotic interstitial pneumonia. Am J Respir Crit Care Med 2005;171:639-44.

19. du Bois RM, Weycker D, Albera C, et al. Forced vital capacity in patients with idiopathic pulmonary fibrosis: test properties and minimal clinically important difference. Am J Respir Crit Care Med 2011;184:1382-9.

20. Biomarkers definitions working group. Biomarkers and surrogate end-points: preferred definitions and conceptual framework. Clin Pharmacol Ther 2001;69:89-95.

21. Grimes DA, Schulz KF. Surrogate end-points in clinical research: hazardous to your health. Obstet Gynecol 2005;105:1114-18. 